\title{
Quantitative Measurement of local shear strain in nano-scale using 3D-ET
}

\author{
Kenji KANEKO *, Koji INOKE ${ }^{* * *}$, Matthew WEYLAND ${ }^{* * *}$, Paul A. MIDGLEY ${ }^{* * *}$, \\ and Zenji HORITA * \\ * Department of Materials Science and Engineering, Faculty of Engineering, Kyushu \\ University, 6-10-1, Hakozaki, Higashi, Fukuoka 812-8581, Japan \\ ** FEI Company Japan Ltd., 13-34, Kohnan 2, Minato, Tokyo, 108-0075, Japan \\ *** Department of Materials Science and Metallurgy, University of Cambridge, \\ Pembroke Street, Cambridge, CB2 3QZ, UK.
}

A number of studies have already shown that the Guinier-Preston (GP) zones have facets parallel to $\{111\}$ planes of the face-centered cubic (fcc) Al-rich matrix [1-4]. Kerry and Nicholson had proposed that GP zones could be sheared by a movement of successive dislocations through the zone [5].

High purity (99.99\%) Al and high purity (99.9\%) Ag were melted in air, cast into an ingot with dimensions of $17 \times 55 \times 120 \mathrm{~mm}^{3}$ and then homogenized at $480{ }^{\circ} \mathrm{C}$ for 24 hours. All samples were solution-treated at $550^{\circ} \mathrm{C}$ for 1 hour and subsequently quenched into iced water. In order to apply large strains to the ingot, and thus to shear GP zones and any $\gamma^{\prime}$ phases, the ECAP (Equal-Channel Angular Pressing) process was chosen [6]. ECAP was conducted at room temperature using a die having a channel angle $\Phi$ of $90^{\circ}$ and with an internal arc of curvature of $\Psi \approx 20^{\circ}$ at the point of intersection of the two channels. It can be shown that the chosen values of $\Phi$ and $\Psi$ lead to an equivalent strain of $\sim 1$ on each passage through the die [7].

$3 \mathrm{D}$ electron tomography (3D-ET) is a method for determining the 3D structure using a tilt series of 2D images. Such 2D images were acquired on a TECNAI F20 field emission gun transmission electron microscope operating at $200 \mathrm{kV}$, with specially designed Fischione 2020 high-tilt holder. After careful alignment, the 3D structure was then reconstructed and volumes rendered by processing a series of $2 \mathrm{D}$ images taken at regular tilt intervals. The each series was acquired from $-70^{\circ}$ to $+70^{\circ}$ with an image every $1^{\circ}$ giving a total of 141 images. A 3D reconstruction was achieved through a weighted back-projection of consecutive 2D slices using IMOD and a volume-rendered image was completed using AMIRA 3.0 [8].

The 2D and 3D-volume rendered images (figure 1(a) and (b)) of the undeformed specimen show the presence of nearly spherical GP zones. In contrast, those of the deformed (ECAP for 1-pass) specimen (figure 1 (c) and (d)) show that the presence of narrow shear bands comprising now ellipsoidal GP zones due to the cutting action of moving dislocations. The average diameter of the undeformed (spheroidal) GP zones is measured as $8.5 \mathrm{~nm}$. The volume of the nearly ellipsoidal GP zones is assumed to be the same as that of the undeformed GP zones, and the average aspect ratio of the ellipsoids is approximately $1: 1: 3$, independent of their absolute size. The angle, $\theta$ between the $<110>$ slip direction and the ellipsoidal line (the major axis of the ellipsoid) was measured as $\sim 30^{\circ}$, and the localized shear strain on the GP zones was therefore calculated as 
$\tan (\pi / 2-\theta)$ i.e. $\sim 1.8 \pm 0.3$. The deformation appears to be highly localized on only a few active slip planes so that only a few highly deformed shear bands develop, as seen in the 2D image and reconstructed 3D volume taken from the 1-pass specimen (Fig 1(c) and (d)).

\section{References}

[1] Gragg J. E. \& Cohen J. B., Acta Metall. 19, 507-519 (1971)

[2]. Dubey Ph. A., Schonfeld B. \& Kostorz G., Acta Metal. Mater. 39, 1161-1170 (1991)

[3]. Gragg J. E. \& Cohen J. B., Acta Metall. 19, 507-519 (1971)

[4]. Malik A., Schönfeld B., Kostorz G. \& Pedersen J. S., Acta Mater. 44, 4845-4852 (1996)

[5]. Kelly A. \& Nicholson R. B., Prog. Mater. Sci. 10, 151-391 (1963)

[6]. Segal V. M., Reznikov V. I., Drobyshevskiy A. E. \& Kopylov V. I., Izvestiya AN SSSR: Metally 1, 99-105 (1981)

[7]. Iwahashi Y., Wang J., Horita Z., Nemoto M. \& Langdon T. G., Scripta. Mater. 35, 143-146 (1996)

[8]. Kremer J. R., Mastronarde D. N. \& Mcintosh J. R., J. Struct. Bio. 116, 71-76 (1996)
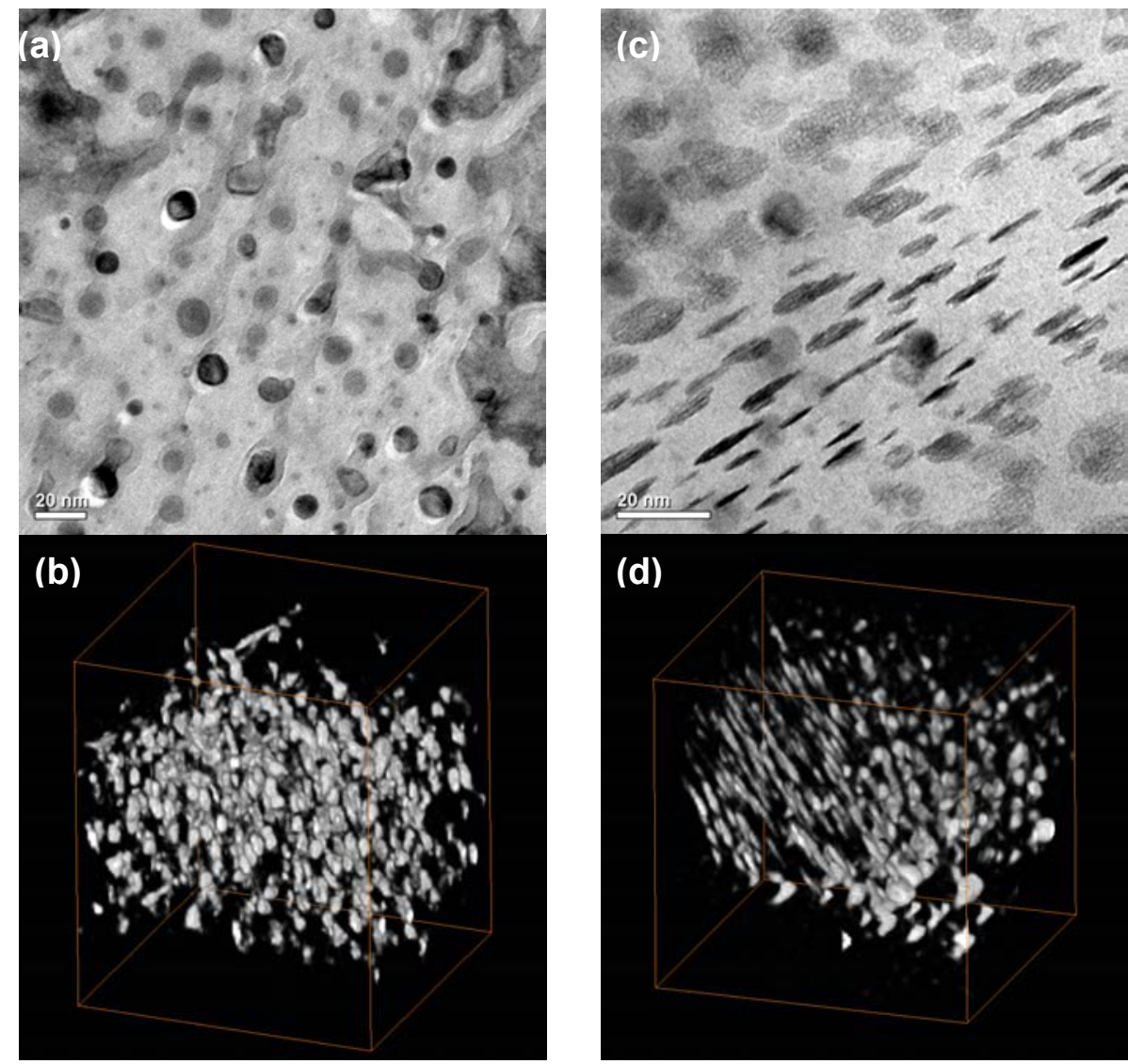

Fig 1 (a) 2D images and (b) reconstructed 3D volume for undeformed specimen, and (c) 2D images and (d) reconstructed 3D volume for ECAP 1-pass specimen. 